\title{
Corrigendum: Protocadherin Celsr3 is crucial in axonal tract development
}

Fadel Tissir, Isabelle Bar, Yves Jossin, \& André M Goffinet

Nat. Neurosci. 8, 451-457 (2005)

In the print version of this article and the version initially published online, an author name was omitted. The fourth author should have been listed as Olivier De Backer of the Molecular Physiology Research Unit, University of Namur Medical School, 61, rue de Bruxelles, B5000 Namur, Belgium. The error has been corrected in the HTML and PDF versions of the article. This correction has been appended to the PDF version. The authors regret the error.

\section{Corrigendum: Ryk-mediated Wnt repulsion regulates posterior-directed growth of corticospinal tract}

Yaobo Liu, Jun Shi, Chin-Chun Lu, Zheng-Bei Wang, Anna I Lyuksyutova, Xuejun Song \& Yimin Zou

Nat. Neurosci. 8, 1151-1159 (2005)

In the print version of this article and the version initially published online, one author's name was spelled incorrectly. The correct spelling is Xue-Jun Song. The error has been corrected in the HTML and PDF versions of the article. This correction has been appended to the PDF version. The authors regret the error.

Corrigendum: Activity-dependent decrease of excitability in rat hippocampal neurons through increases in $I_{h}$

Yuan Fan, Desdemona Fricker, Darrin H Brager, Xixi Chen, Hui-Chen Lu, Raymond A Chitwood \& Daniel Johnston

Nat. Neurosci. 8, 1542-1551 (2005)

In the print version of this article and the version initially published online, the units for anisomycin concentration in the figure labels for Fig. $8 \mathrm{~d}$ and $\mathrm{f}$ were incorrect. The correct concentration is $20 \mu \mathrm{M}$. The error has been corrected in the HTML and PDF versions of the article. This correction has been appended to the PDF version. The authors regret the error. 